\title{
Pensar la sociedad: temas, problemas, retos para las ciencias sociales
}

\author{
Ximena Valdés S. \\ CEDEM, Santiago, Chile. \\ Email: ximena.valdes@cedem.cl
}

\begin{abstract}
Resumen: Estas notas realizan un rápido recorrido a las preguntas planteadas por las ciencias sociales y humanidades, revisando los cambios en las instituciones que las cobijaron, los temas y problemas que se plantearon, la relación entre producción de conocimientos, cambio social, modernización, cultura y medio ambiente, a lo largo de las últimas décadas y, por último, se plantean algunas de las dudas que merecen los nuevos formatos estandarizados de medición de resultados en la producción de conocimientos a los que ha adscrito nuestro país y sus instituciones científicas y universidades.
\end{abstract}

Palabras clave: ciencias sociales, nuevos temas y problemas, cambios institucionales.

\section{Think of society: issues, problems, challenges for the social sciences}

\begin{abstract}
These notes carry a quick tour to the questions raised by the social sciences and humanities, reviewing changes in the institutions that sheltered, issues and problems encountered, the relationship between social knowledge production change, modernization, culture, environment over recent decades and, finally, raises some new questions that deserve standardized formats for measuring results in the production of knowledge that has been assigned to our country and its scientific institutions and universities.
\end{abstract}

Keywords: social sciences, new issues and problems, institutional changes

\section{Pensar a sociedade: temáticas, problemas, desafios para as ciências sociais}

Resumo: Estas notasrealizam umrápido percursoàs questões levantadaspelas ciênciassociais e humanas, revendo as mudanças nasinstituiçõesque as abrigaram, questões e problemas encontrados, a relação entre a produção de conhecimento, mudançasocial, modernização, cultura emeio ambientenas últimas décadase, finalmente, levanta algumasdas duvidas que merecemos novos formatos padronizadospara medir os resultadosna produção deconhecimentoque foram atribuídosao nosso paíse suas instituiçõescientíficase universidades.

Palavras-chave: ciências sociais, novas temáticas e problemas, mudanças institucionais. 
El llamado de la Revista Polis a la reflexión sobre lo que han sido y son las Ciencias Sociales latinoamericanas, se puede enfocar a partir de lo que Michel de Certeau denomina “el lugar de producción”. Este es un punto de partida que posibilita comprender el modo cómo se produjeron las ideas, desde dónde se hablaba, la institucionalidad que sostenía la producción intelectual en Ciencias Sociales.

¿Qué interrogantes animaron a los cientistas sociales a mediados del siglo XX? ¿Bajo qué tipo de institución desarrollaron sus ideas, sus investigaciones y a qué sirvieron los resultados? ¿Cómo han variado temas y problemas hasta ahora? ${ }^{1}$

En segundo término este artículo trata de colocar en común las dudas y preocupaciones sobre las políticas de desarrollo científico que se realizan en Chile ${ }^{2}$ en el marco de la privatización de la educación superior que arrancó con la dictadura militar, de la política científica y de sistemas de evaluación en la producción de conocimientos establecidos como reglas de productividad.

\section{Instalaciones, desplazamientos e institucionalidad}

A lo menos cuatro períodos marcan el trayecto de las Ciencias Sociales en Chile y Latinoamérica. Las disciplinas reunidas en lo que llamamos Ciencias Sociales, son de aparición relativamente reciente. La Sociología, Antropología, Ciencia Política, etc., son mucho más tardías que el Derecho y las Ciencias Jurídicas y, desde luego, la Historia.

Hasta la mitad del siglo XX la reflexión sobre la sociedad, sus problemas y soluciones, se realizó generalmente en formato de ensayo, desde el Derecho, las Ciencias Jurídicas y las Humanidades. No es por casualidad que esto ocurriera de este modo ya que el asunto central que preocupaba a los intelectuales y políticos era la construcción del Estado moderno. Para los juristas se trataba de comprender el modo cómo debería funcionar el Estado en una sociedad que se acercaba o quería acercarse a la modernidad. Los historiadores por su parte construían más la Historia del Estado, que de la sociedad, en el marco de una institucionalidad republicana todavía joven. Es necesario notar que fueron los extranjeros que llegaban a Chile por ejemplo quienes analizaban y se interesaban en la sociedad, en los grupos sociales marginales, los indígenas, etc...

Durante la primera mitad del siglo XX, estas disciplinas tenían por referente más que a la sociedad, la relación entre Estado y sociedad. Se pensó en esos años en cómo construir la sociedad industrial y en el papel que debía jugar el Estado en dicha empresa ${ }^{3}$. A la "cuestión social” que se venía arrastrando desde el siglo XIX como problema se la encaró otorgando un papel central el Estado. Figuras como Arturo Alessandri tomaron como tema de sus Tesis de Grado la vivienda obrera mientras el salario familiar obrero fue desarrollado por Eduardo Frei Montalva, es decir, la 
habitabilidad de la fuerza de trabajo y las formas de remuneración a la clase obrera se yerguen como campos que serán objeto de intervención y regulación pública.

El "lugar de producción" por lo general fueron las Tesis universitarias en las Escuelas de Derecho y posteriormente los libros de ensayo político que marcaron la intelectualidad política de la primera mitad del siglo XX chileno. En este sentido las editoriales ligadas a los Partidos Políticos jugaron un papel central, como Editorial del Pacífico, Prensa Latinoamericana y otras. Las revistas de mayor prestigio y dónde se desplegó el conocimiento social fueron también publicaciones ligadas a los Partidos Políticos, como Política y Espíritu a la Falange Nacional, Principios al Partido Comunista, Arauco al Socialista, Lircay a los nacionalistas, o a instituciones universitarias como el Boletín de la Universidad de Chile, Finis Terrae a la Universidad Católica, etc...

Un asunto diferente y de sumo interés en la historia de la producción intelectual chilena son las revistas ligadas a la Biblioteca Nacional, Revista Mapocho, o instituciones como la Sociedad chilena de Historia y Geografía, cuya Revista es quizá la de mayor relevancia intelectual en todo el período.

Estas producciones estuvieron marcadas por el evolucionismo histórico y el liberalismo político. La impronta de historiadores como Diego Barros Arana será duradera hasta muy entrado el siglo xx. El prestigio de la "Historia”, como la disciplina mayor de las Ciencias Sociales, vendrá quizá del papel jugado por estos intelectuales en la construcción del "relato de La Nación”. Jaime Eyzaguirre desde posiciones de derecha, por ejemplo, en sus clases memorables de la Facultad de Derecho tanto de la Universidad de Chile como de la Católica, enhebraba la Historia y el Derecho otorgándole a los nuevos abogados un aura de misioneros poco menos del Estado y la Nación y a la política un sentido de vocación sagrada.

Un segundo espacio de producción de lo que eran en ese momento las Ciencias Sociales estuvo dado por las nacientes escuelas de Servicio Social. No es por casualidad que estas se desarrollaron arrimadas a las Escuelas de Derecho.

La Asistencia Social ${ }^{4}$ repartida entre las universidades Católica y de Chile, pensó la salud del pueblo y a la familia popular del campo y la ciudad acorde con el progreso de la industrialización del país, asignando lugares y funciones en los repartos de género propios de la época: hombres en el trabajo obteniendo un salario para mantener a la familia (que debía sacársela del "desorden" que arrastraba desde el siglo XIX); mujeres en lo doméstico a cargo de la reproducción social y biológica de los hijos. Todo desvío del orden de género industrial fue sancionado en un campo profesional que sería uno de los dispositivos fundamentales para encarar "lo social" en universidades, instituciones públicas y también eclesiásticas. Los discursos de las entonces Asistentes Sociales -laicas y católicas- sorprenden por su similitud con respecto a la afirmación de la familia moderno-industrial, 
conyugal, en la conceptualización de Durkheim. Se desestimuló desde la Asistencia Social -profesión por esencia bisagra entre familia y Estado- el trabajo femenino fuera del hogar aludiendo a que este, perjudicaba los preceptos de buena madre y esposa y contribuía a que las mujeres desobedecieran a la autoridad masculina en el hogar y descuidaran la adecuada crianza de su prole ${ }^{5}$. Fue el período donde se pensó -en Chile, como en otros países- desde la lógica de “ordenar la sociedad” combatiendo, a través de un corpus de ideas propias del período de afirmación de la sociedad industrial, el desorden legado por la sociedad tradicional del siglo XIX a la sociedad moderno-industrial ${ }^{6}$.

Las preguntas centrales, antes que las ciencias sociales alcanzaran su status científico giraron en torno a cómo construir un tipo de sociedad que se iba desprendiendo de un pasado rural con todas sus consecuencias (migraciones campo-ciudad, miseria urbana, proliferación de conventillos, desnutrición, mortalidad infantil y así en adelante). Las reflexiones se movieron en ese entonces entre la caracterización, naturaleza y la función del Estado y los problemas que enfrentaba una sociedad que se urbanizaba e industrializaba, en un contexto de la articulación entre la producción de conocimientos en universidades, y el Estado en tanto agente de transformación de lo social.

En Chile no florecieron las disciplinas que complejizaban la sociedad, los individuos o percibían males sociales fuera del ámbito del Estado y el derecho, tales como la psicología, antropología. Estas surgieron tímidamente marginales a las Universidades, en Hospitales Psiquiátricos o Museos y no pocas veces, fueron extranjeros quienes las desarrollaron.

\section{Domicilios: universidades e instituciones latinoamericanas}

Una segunda etapa correspondería al período de institucionalización de las ciencias sociales al interior del campo universitario. Se trata de un momento de profesionalización de muchas de las disciplinas de las Ciencias Sociales en el continente, especialmente la Sociología (José Medina Echavarría en México, Gino Germani, en Buenos Aires). Esta disciplina en particular, además de avanzar en métodos y técnicas que la constituían dentro del campo del saber científico, desde mediados del siglo XX, tuvo como horizonte preguntarse por los problemas derivados de la modernización, de la urbanización y la marginalidad, la cuestión del desarrollo aportando en los debates sobre el desarrollismo, la dependencia, el populismo, entre otros muchos y, desde luego, con variadas interpretaciones y al alero de distintos espacios institucionales (en el caso chileno, CEPAL (Cardoso y Faletto), CESO/Universidad de Chile (André Gunder Frank, Ruy Mauro Marini), CEREN/Universidad Católica (Armand Mattelart, Manuel Antonio Garretón, Franz Hinkelammert, Tomás Moulián, entre otros).

En las décadas del sesenta del siglo veinte, se produjo un importante desarrollo de las Ciencias Sociales por cuanto distintas disciplinas, domici- 
liadas en universidades y Centros de Estudio de alcance latinoamericano, extendieron sus propuestas docentes y de investigación a la formación de cientistas sociales de todo el continente, en sedes de instituciones como la FLACSO en Chile, ESCOLATINA en la formación de economistas y sobre todo el programa ILPES de la CEPAL, formador de una gran cantidad de profesionales de la Planificación Social como se denominaba en la época.

Durante este período, al situar el foco en la producción de conocimientos en una etapa en que hubo una de las políticas públicas más importantes de la última fase del período de sustitución de importaciones (las reformas agrarias), podemos afirmar que los estudios principalmente de los geógrafos en Chile (sociólogos/antropólogos como Stavenhagen en México), fueron los que sirvieron de argumento y fundamento, en el contexto de un arduo debate público y político sobre el monopolio de la tierra por parte de la elite oligárquica a una política pública orientada a la democratización de la sociedad rural (redistribución de la propiedad de la tierra y legislación sindical). Rafael Baraona y su equipo, Gene Ellis Martin, Jean Borde (y Góngora), fueron clave en los debates sobre la necesidad de la reforma agraria que, en toda América Latina, sucedió a los Informes CIDA de cada país en el marco del cumplimiento de la agenda fijada por la Alianza para el Progreso para el sub-continente latinoamericano ${ }^{7}$. En medio de este proceso, la producción de conocimientos sobre el devenir del reparto de tierra a campesinos muestra cómo las Ciencias Sociales, al igual que en el período inicial del "desarrollismo" y de institucionalización de una disciplina como la sociología (Gino Germani, UBA) y de su desarrollo posterior (Galtung, Touraine, Faletto, etc. en FLACSO/CHILE)- estaba muy enlazada a los procesos de cambio social y desarrollo.

\section{Diáspora de intelectuales y fuga de las ciencias sociales a espacios protegidos}

Estas orientaciones y desarrollos tocaron a su fin con el golpe de Estado de $1973^{8}$ que clausuró las Escuelas de Sociología en particular y Centros como CEREN y CESO de las universidades Católica y de Chile respectivamente; exilió y persiguió a cientistas sociales, limitando la producción de conocimientos que hasta entonces se había desplegado en los espacios universitarios. De esta forma se abre un tercer período de refugio de las Ciencias Sociales en espacios protegidos de la intervención militar, lugares no universitarios al alero de la iglesia católica -Academia de Humanismo Cristiano- ONGs y en Centros de estudios como FLACSO (que había albergado a la ELAS, Escuela Latinoamericana de Ciencias Sociales) acogiendo a estudiantes y profesores de todo el continente previamente exiliados de sus países, por otras dictaduras, especialmente la brasileña.

Este período se caracteriza también por los cambios de paradigmas en las Ciencias Sociales en particular por la crisis del pensamiento marxista tradicional. Surge un pensamiento mucho más ecléctico en que los cientistas 
sociales van a buscar datos empíricos que explican las dificultades por las que atraviesan sus países.

En el nuevo contexto dictatorial, surgieron nuevos temas y problemas. Hubo un desplazamiento desde las preguntas a la modernización y el desarrollo - grosso modo- a la revalorización de la democracia, los sistemas políticos, los derechos humanos y la democratización de sociedades cautivas de regímenes militares (Norbert Lechner, por ejemplo). No obstante la importancia que adquirieron los debates sobre la democracia, en los ochenta ante la crisis de los sistemas de representación, emergieron nuevos temas y problemas como resultado de la presencia y expresión pública de los llamados nuevos movimientos sociales ${ }^{9}$ (de pobladores, de indígenas, de mujeres y feminista). La cuestión étnica, las desigualdades por razones de sexo/género se hicieron presentes en la nueva tematización de las ciencias sociales incorporando conceptos y dimensiones de análisis de la realidad social ausentes hasta entonces, dadas las concepciones sobre la uniformidad y homogeneidad de la sociedad, de la irrelevancia que para las Ciencias Sociales tuvo la desigualdad por diferencia sexual, étnica, racial. Durante este período hubo entonces un volcamiento "hacia adentro", en espacios protegidos procurando, con todo, mantener la producción de conocimientos. En este contexto se sumó además la "nueva historia” que cuestionó un tipo de discurso histórico propio de la historia oficial con importantes aportes en el campo de la historia del "bajo pueblo"10 y de los grupos subalternos, la historia de las mujeres, de la opresión y la desigualdad sexual ${ }^{11}$, de los indígenas ${ }^{12}$, de los pobladores ${ }^{13}$.

En estos espacios no-gubernamentales y fuera de lo "académico formal”, problemas candentes como la pobreza, el desempleo y la cesantía, la reestructuración económica causada por la aplicación de las políticas de ajuste, la pérdida de recursos e instancias de organización de los trabajadores, la feminización de los mercados laborales, la ruptura de la familia de la sociedad salarial, van a constituir respuestas de las ciencias sociales a los nuevos problemas surgidos de la apuesta al paradigma neoliberal y de los cambios culturales inducidos por la globalización.

\section{El retorno a un nuevo escenario universitario}

Un cuarto período se abre con la recuperación de la democracia. Desde los noventa las ciencias sociales transitaron desde los espacios protegidos a su re-instalación en universidades (que ya eran muchas sumando públicas y privadas tradicionales a las nuevas privadas) sin que este tránsito implicara un auge notorio en la producción de conocimientos en los centros de estudios de educación superior ${ }^{14}$. Tras el encapsulamiento producido por la dictadura que se abrió una vez que se recuperó la democracia, los procesos de redemocratización en las universidades para recuperarse tal como lo habían sido hasta antes del golpe de Estado, sedes y productoras del pensamiento de lo social, fueron lentos, leves y parciales. Se anudó en su gestión a partir de los años noventa la permanencia del 
“antiguo régimen” (que había cercenado el desarrollo de las ciencias sociales) y nuevos y antiguos aspirantes de ingreso y regreso al mundo académico con grandes dificultades de entrada a las generaciones más jóvenes.

El campo universitario había cambiado radicalmente: se desestructuró (caso de las sedes regionales de la Universidad de Chile), se privatizó (universidades privadas dentro de las cuales muchas de ellas ligadas a órdenes religiosas -Opus Dei, Legionarios de Cristo, Salesianos, Jesuitas y/o a grupos económicos y cadenas de educación superior internacionales-, constituyéndose un nuevo campo de negocio acorde con el papel subsidiario del Estado, la continuidad del paradigma neoliberal y el proceso de mundialización de fines del siglo $\mathrm{XX}^{15}$.

Bajo esta nueva situación, las Ciencias Sociales que habían germinado originalmente en las pocas universidades que existían hasta los años setenta (Universidad de Chile, Católica, Universidad de Concepción) se dispersaron entre las primeras y algunas de las nuevas universidades privadas. Ello produjo una importante atomización y fragmentación que no se tradujo en un declive de las Ciencias Sociales ya que proliferaron temas y problemas acordes tanto al perfil ideológico de las diversas casas de estudio como de las preguntas y nuevas interrogantes que colocó la llamada post-modernidad y/o capitalismo tardío, dentro de las cuales, los temas culturales que comenzaron a abundar en paralelo con aquellos que hurgaron en el mantenimiento de la vulnerabilidad producto de la desprotección social.

Ciertas preguntas se mantuvieron desde los años ochenta como las vinculadas a la pobreza ${ }^{16}$, a la transición democrática, la des-sindicalización de los trabajadores, la flexibilidad laboral. Dentro de las nuevas preguntas que continuaron interpelando al modelo económico y sus consecuencias sobre las poblaciones y trabajadores/as, se agregaron aquellas que interpelaron al patrón hegemónico de familia, de masculinidad y femineidad. El referente igualdad de género continuó abordándose desde distintas disciplinas, la sexualidad junto al tema de la soberanía del cuerpo/de los cuerpos se instala, la libre opción sexual, la homofobia, la homosexualidad, la discriminación por razones de sexo, etnia y raza, las migraciones y su racialización serán los nuevos temas y objetos de estudio de las ciencias sociales que abren el nuevo siglo y forman parte tanto de los problemas y ventajas que acarrea la mundialización como de los límites que impone el modelo económico sobre los trabajadores de ambos sexos y sobre otras categorías sociales (campesinos, clases medias...). A estos nuevos temas y problemas se agrega resultado de los efectos del modelo primario-exportador, el tema ambiental y sus consecuencias sobre territorios y poblaciones. En términos institucionales cabe afirmar que en algunos temas, los Centros Privados de Investigación no son necesariamente reemplazados por las universidades en materia de investigación y productividad, especialmente en los estudios que consideran la variable género. En términos de autores hay que señalar que en la "cuestión indígena" hay cambios que se observan a nivel editorial, con el surgimiento de intelectuales y autores indígenas, fenómeno no exclusivo a Chile ${ }^{17}$. 
En este contexto ha surgido la pregunta sobre la perdurabilidad del carácter latinoamericano que alcanzaron las ciencias sociales desarrolladas en universidades y organismos como FLACSO y CLACSO, en el contexto de la globalización. Pregunta planteada por Stavenhagen en el 35 aniversario de FLACSO México a la que intenta responder este llamado de Polis a la reflexión a la que algunos autores y corrientes han respondido con un tipo de producción de conocimientos "re-situados": los del "sur"18.

\section{De cara a la medición de la productividad.}

Uno de los problemas y desafíos de las ciencias sociales inmersas en un mundo globalizado que impone y se autoimpone estándares de productividad es la situación de la validación de los resultados de investigación en las distintas categorías de revistas indexadas desde las ISI, SCOPUS, SCIELO.... y de la devaluación del "artefacto" libro que iluminó con ideas hasta hace poco a la humanidad y, desde luego, a nuestro continente.

Una hipótesis plausible es que las ciencias sociales tienden progresivamente a desprenderse de sus vínculos con sus propias sociedades, que se ha debilitado su incidencia en lo social, en las políticas públicas, en el desarrollo, en la crítica a las consecuencias que acarrea el nuevo modelo de acumulación y los enormes cambios culturales y medio-ambientales producidos y vehiculizados por la misma globalización.

A esto se suma la privatización de las entidades que están al origen de formación de investigadores, de cientistas sociales e intelectuales. La desterritorialización del saber y de la formación académica (desplazamiento de lo público a lo privado, de lo nacional a lo internacional) así como de la producción de conocimientos parece haber hecho necesario el arbitraje bajo parámetros externos que en buena medida encuentran una explicación plausible en que, habiendo perdido la incidencia de las ciencias sociales en la sociedad que habitan -la pregunta del para qué de las ciencias sociales, es decir, su sentido- se juzga, mide y evalúa bajo indicadores creados en el mundo anglo-sajón que, a la vez que globaliza el campo lo tiende a hacer autoreferido (cuánto se cita tal autor para medición de impacto no cómo esta u otra investigación contribuyó a la modificación del actuar del Estado, de las políticas públicas, la democracia, la calidad de vida de determinado sector, en la difusión y debate de ideas, etc.) como parte de una estrategia internacionalizada de un grupo de investigadores que usualmente se leen y citan a sí mismos en determinados ámbitos editoriales como las revistas ISI $^{19}$.

En Chile, el Consejo Nacional de Ciencia y Tecnología ha hecho suyos estos parámetros de productividad en sus programas estableciendo los mismos criterios para las ciencias duras con las ciencias sociales y humanidades (FONDECYT, FONDAP, Milenio, Anillos, etc.) De otra parte, la política nacional de formación de capital avanzado ha sido agresiva en crear un sistema de becas e incentivos bajo la modalidad de concursos para 
la formación en los grados de Magister, Doctorado y post-Doctorado bajo evaluación curricular con semejantes parámetros. No está claro que haya racionalidad entre la adquisición del grado de Doctor/a, el número de Doctores/as y la empleabilidad puesto que el mismo sistema previsional y de remuneraciones universitarias evita que los profesores jubilen a una edad adecuada abriendo plazas a los/as más jóvenes. Frente a esta situación, y quizás para mitigarla, CONICYT ha creado distintos programas para fomentar el desarrollo de las ciencias, entre ellas en un lugar menor, de las ciencias sociales (programa FONDECYT Regular e Iniciación y concursos post-doctorales, Anillos, Milenio, FONDAP), en líneas prioritarias de investigación o incentivando la formación de redes entre distintas universidades y centros de estudio. Se coloca en sus requerimientos la incorporación de jóvenes doctorantes y post-doctorantes abriendo camino a la inserción de éstos en esas instituciones que articulan investigadores de distintas universidades. Esta podría ser una iniciativa útil para mitigar la fragmentación y dispersión de las Ciencias Sociales en distintas universidades puesto que dichos programas buscan articular temas e investigadores/as no obstante también tiende a concentrar los recursos y a privilegiar ciertos temas y no otros acentuando los mismos sistemas de evaluación de productividad bajo normas de “indexación”.

Este nuevo sistema de control de las Ciencias Sociales tiende a constituir un "campo" regulado que empuja a que los académicos operen de manera políticamente neutral. Es una tendencia a separar el campo intelectual del campo académico. La obligación antedicha de ingresar a redes globalizadas conlleva la pérdida del carácter intelectual en el sentido sartriano del término y la adopción de un estilo de trabajo institucionalizado.

\section{Pensando en los desafíos a futuro}

No obstante la pertinencia de esta pregunta al considerar la universalidad de los fenómenos y procesos que acarrea la mundi/globalización sobre nuestra y otras sociedades, hay temas y problemas que continúan desafiando el papel y el lugar de las Ciencias Sociales en nuestro país y Latinoamérica. Pero antes que los temas y problemas un desafío es situarse en la ruptura de las fronteras disciplinarias para lograr, a través de estudios multidisciplinarios comprender la complejidad de los fenómenos sociales contemporáneos.

En segundo lugar, someter el campo a la pregunta ¿para qué las ciencias sociales? ¿es posible pensar en el desarrollo -como ocurrió en los sesenta- con el papel de los Estados debilitados como están frente a un mercado que hegemoniza el devenir de lo social? ¿Escuchan, leen, miran, los gestores de las políticas públicas los debates sobre los diversos problemas que acumula la sociedad y, en particular, los que recaen en los sectores más desfavorecidos y vulnerables? Al parecer, poco y nada ${ }^{20}$ lo que plantea la duda sobre la incidencia de las Ciencias Sociales en el cambio social. Si parece ser el caso de los movimientos sociales bajo la dictadura -que 
generaron una nueva institucionalidad pública y políticas inclusivas, y de los movimientos post-democratización ${ }^{21}$ que han gatillado reformas en el campo tributario y de la educación.

En tercer lugar, temas del mundo presente que se reflejan en las Tesis de Grado y Post-grado como los culturales (desigualdad y discriminación de género, masculinidad, homofobia, homosexualidad, transexualidad, etnicidad...) de hecho contribuyen a generar ideas y debates que contribuyen a la democratización de nuestras sociedades pero es necesario recordar que han sido los propios actores quienes los han colocado en la arena pública antes que los cientistas sociales y que, paradojalmente, no necesariamente han permeado las distintas disciplinas y programas docentes[22.

A estos nuevos temas de las ciencias sociales contemporáneas se agregan aquellos que generan conocimientos sobre procesos migratorios de nuestros países, tanto intra-nacionales como transfronterizos e internacionales. Los vínculos de los "parias” del mundo globalizado como parecen ser los migrantes con la marca de género, etnia y raza son estrechos ${ }^{23}$ y conforman una nueva cara de la actual “cuestión social” ya que están articulados, imbricados con los fenómenos de flexibilidad laboral y precarización de los mercados de trabajo. La pregunta ¿Dónde y cómo trabajan los migrantes -mujeres, indígenas, extranjeros- es pertinente para las ciencias sociales contemporáneas. Así como éste, la violencia podría constituir un campo de investigación privilegiado (en estadios, en la calle, en las manifestaciones públicas, en la casa y la pareja).

El tránsito de los estudios sobre la pobreza a los estudios sobre la desigualdad es importante por hurgar con mayor profundidad en las raíces de este fenómeno tan visitado en los años noventa sin atender necesariamente a sus causas ni expresiones.

Sin embargo, más allá de los temas y problemas, muchas de las preguntas que dieron su sentido a las ciencias sociales permanecen en un área de incertidumbre.

¿Podemos hoy, desde las ciencias sociales, hablar de "desarrollo" como lo hicieron los cepalianos de los años sesenta? Este concepto atraviesa por una de sus más complejas encrucijadas enraizadas en el debilitamiento del papel del Estado y la fortaleza que adquirió el mercado en la toma de decisiones sobre las poblaciones, los territorios ${ }^{24}$, el medio ambiente, las naciones hoy diluidas en "los espacios del capital"25.

Frente a modelos de desarrollo extractivistas, las poblaciones afectadas junto al medio ambiente crecientemente deteriorado por las inversiones mineras y de generación de energía constituyen nuevos temas y problemas para las ciencias sociales. Pero también las ciencias sociales encaran grandes desafíos en el plano de la cultura y la afirmación de identidades particulares, temas que surgieron al calor de movimientos sociales y de nuevos referentes en que la igualdad ocupa un lugar central. 
¿Podrán las ciencias sociales moverse entre sociedad, política y cultura apuntando a un nuevo paradigma que permita comprender el mundo contemporáneo?

¿Podrán las ciencias sociales desarrollarse frente a este aumento de la complejidad de los problemas del presente bajo los marcos institucionales estandarizados que se les imponen?

¿O deberán liberarse de los constreñimientos institucionales con nuevas estrategias para incidir en las transformaciones que reclama una sociedad cada día más compleja y diversa? 
Polis, Revista Latinoamericana, Volumen 14, $N^{\circ}$ 41, 2015

\section{Notas}

${ }^{1}$ Manuel Antonio Garretón en el libro Las ciencias sociales en la trama de Chile y América Latina. Estudio sobre las transformaciones sociopolíticas y movimiento social (2014), LOM, Santiago, ha analizado en la primera parte del libro la trayectoria y perspectiva de las ciencias sociales en Chile.

${ }^{2}$ Nos referimos a la política de desarrollo de las Ciencias Sociales vigente en la Comisión Nacional de Ciencia y Tecnología -CONICYT- y su Programa Fondecyt, Anillos, Milenio, Fondap, Programa de formación de capital humano avanzado a través del sistema de becas de post-grado (Magister, doctorales y post doctorales) dentro y fuera del país.

${ }^{3}$ Arturo Alessandri (1883) Habitaciones obreras, Memoria Escuela de Derecho de la Universidad de Chile para obtener el Título de Abogado al igual que Eduardo Frei Montalva en la misma Escuela de Derecho (1933) El régimen del salariado y su posible abolición, Editorial del Pacífico, Santiago.

${ }^{4}$ María Angélica Illanes (1993) En el nombre del pueblo, el Estado y la Ciencia. Historia Social de la Salud Pública. Chile 1880-1973. Colectivo Atención Primaria, Santiago; (2004). Cuerpo y sangre de la política. La construcción histórica de las Visitadoras Sociales (1887-1940). LOM, Santiago.

${ }^{5}$ Ximena Valdés (2007) La vida en común. Familia y vida privada en Chile y el medio rural en la segunda mitad del siglo XX. LOM, Santiago.

${ }^{6}$ Jacques Donzelot (1994) La policía de las familias. Pre-textos, Valencia; Michel Foucault (1997) Il faut défendre la société. Cours College de France 1976. Seuil, Paris

${ }^{7}$ Roberto Santana (1996) Agricultura chilena en el siglo XX: contexto, actores y espacios agrícolas. DIBAM, Santiago.

${ }^{8}$ El golpe de Estado brasilero empujó a intelectuales de ese país a radicarse en Chile como ya había ocurrido con anteriores dictaduras, especialmente centroamericanas que impulsaron el exilio de intelectuales hacia los países del sur del continente.

${ }^{9}$ Manuel Antonio Garretón (2014), op. Cit. Pág. 216.

${ }^{10}$ ] Gabriel Salazar. Labradores, peones y proletarios (1984). SUR Ediciones, posteriormente varias ediciones en LOM.

${ }^{11}$ En especial la producción de Julieta Kirwood en FLACSO, Elizabeth Hutchison, Alejandra Brito, Soledad Zárate (véase Disciplina y desacato. Construcción de identidad en Chile, siglos XIX y XX (1994) SUR/CEDEM, Santiago; Cecilia Salinas, La mujer Proletaria, una historia por contar (1987), LAR, Santiago; Claudia Rojas, Edda Gaviola, Lorena Lopresti, Ximena Jules. (2007) Queremos votar en las próximas elecciones, LOM, Santiago.

12 José Bengoa (1996, $3^{\text {a }}$ edición), Historia del pueblo mapuche. SUR Ediciones y luego varias ediciones en LOM. 
${ }^{13}$ Por ejemplo, Mario Garcés (2002). Tomando su sitio, LOM, Santiago.

${ }^{14}$ De hecho, la sede de los estudios de género más importantes continuaron siendo los Centros privados de Investigación (Corporaciones, Fundaciones, ONGs).

${ }^{15}$ cfr. María Olivia Monckeberg (2005) La privatización de las universidades. Una historia de dinero, poder e influencias. La Copa Rota, Santiago; Con fines de lucro (2013), Random House Mondadori, Santiago.

${ }^{16}$ Este tema se mantiene por largo tiempo, sin necesariamente profundizar en el análisis de sus causas; se ve como indicador para medir el "estado de la sociedad" en materia de ingresos, como problema para generar políticas públicas pero los sujetos son subsumidos a la categoría de pobres bajo la mirada a la pobreza amputada de sus localizaciones, de su historia, de la identidad de los sujetos, de su inserción en la estructura social: son solamente "pobres" sin nombre hasta que la pobreza "pasa de moda” dando lugar a los estudios de desigualdad social.

${ }_{17}$ Véase por ejemplo los títulos de autores mapuche en la editorial LOM y Claudia Zapata Silva (2013), Intelectuales indígenas en Ecuador, Bolivia y Chile. Diferencia, colonialismo y anticolonialismo. Ediciones Abya-Yala, Serie Pensamiento Americano.

${ }^{18}$ De Latinoamérica al sur: véase Boaventura de Souza Santos, Sociología Jurídica Crítica. Para un nuevo sentido común en el Derecho. En clave de Sur (2009), ILSA, Bogotá; Una Epistemología del SUR (2009), CLACSO/Siglo XXI México.

${ }^{19}$ Se le ha otorgado en España el Premio Príncipe de Asturias a Saskia Sassen. Un fragmento del comentario aparecido en la prensa española (Diario El País, 14 junio 2013) a propósito de esta importante intelectual dice lo siguiente: "La tercera enseñanza de Saskia Sassen nos lleva a la futura quiebra de nuestro sistema académicouniversitario. Una de las científicas más importantes de nuestra época no ha conseguido ningún sexenio, ninguna acreditación, frente a los criterios de nuestras agencias de evaluación, que anteponen siempre el mismo criterio: tres publicaciones JCR (Journal Citation Reports) en los últimos cinco años. Sassen no tiene ni una, sino que ha publicado libros e informes, fruto de proyectos de investigación de verdad y referencias fundamentales para académicos comprometidos, ha publicado numerosos artículos en medios de gran difusión, etc., pero se ha resistido a la práctica de inflar su currículum con artículos estandarizados sin interés ni lectores, más allá de círculos de amigos de citación mutua. Estamos entregando nuestra calidad científica a Thompson Reuters (la empresa gestora de los JCRs) igual que la calificación de nuestras economías a Fitch, Moody's y Standard \& Poor's. La estandarización de nuestra enseñanza universitaria y de nuestra producción científica nos llevará a universidades sin debates, investigaciones sin compromiso y un sistema académico sin pensamiento.El tan merecido premio para Saskia Sassen nos deja, por lo tanto, un sabor agridulce porque puede representar un galardón para una representante de una estirpe en proceso de extinción”.

${ }^{20}$ Teresa Matus, Directora Trabajo Social Universidad de Chile en exposición de Jornadas de FACSO, Universidad de Chile, Mesa 4, Las ciencias sociales y sus implicancias en las políticas públicas, Jornadas de investigación FACSO, Universidad de Chile, 11 julio 2014. 
${ }^{21}$ M.A. Garretón (2014), op. Cit.

${ }^{22}$ Irene Thèry (2004), “Dynamique et égalité de sexe et transformation de la parenté”, en: Femmes, genre et societés, La Découverte, Paris, 2004. Pp.159-166.

${ }^{23}$ Véase trabajos sobre migraciones de extranjeros de María Emilia Tijoux, FACSO, Universidad de Chile; de Carolina Stefoni, Universidad Alberto Hurtado, Sociología.

${ }^{24}$ En los diferentes artículos de Territorios Rurales. Movimientos sociales y desarrollo territorial rural en América Latina (2007), J. Bengoa Ed. RIMISP/Catalonia, Santiago, se evidencian los impactos de las inversiones mineras sobre campesinos e indígenas y también las resistencias que se despliegan frente al deterioro ambiental y la calidad de vida de estas poblaciones. Como ejemplo actual de este problema, las recientesprotestas en el sur de Perú por la Minera Tia María con impactos negativos sobre los campesinos de la zona son elocuentes como formas de resistencia al capital minero.

${ }^{25}$ Davis Harvey (2001), Espacios del capital. Hacia una geografía crítica. Akal, Madrid.

Recibido: 10.08.2015

Aceptado: 25.08.2015 